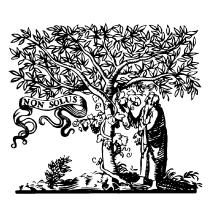

ELSEVIER

\title{
Elastic waves in visco-elastic half-space generated by various vehicle loads
}

\author{
H.-H. Hung, Y.-B. Yang* \\ Department of Civil Engineering, National Taiwan University, Taipei, 10617 Taiwan
}

Accepted 28 September 2000

\begin{abstract}
In this paper, the response of a visco-elastic half-space subjected to moving loads with static and dynamic components is investigated. Four types of vehicle loads are considered, including the moving point load, uniformly distributed wheel load, elastically distributed wheel load, and a train load simulated as a sequence of elastically distributed wheel loads. In each case, the influence of the moving loads traveling in the subsonic, transonic and supersonic ranges on the dynamic responses of the half-space is studied. The parametric study conducted herein enables us to grasp insight into the mechanism of wave propagation for a visco-elastic half-space under moving loads. (C) 2001 Elsevier Science Ltd. All rights reserved.
\end{abstract}

Keywords: Doppler effect; Half space; Mach number; Moving train load; Moving vehicle; Wave propagation

\section{Introduction}

The problem of ground-borne vibrations induced by moving vehicles has been one of increasing interest, partly enhanced by the construction of mass rapid transit systems and high speed railways worldwide. Eason [1] studied the three-dimensional steady-state problem for a uniform halfspace subjected to loads moving with constant speeds, in which both point loads and loads distributed over a circular or rectangular area are considered. The governing equations were solved by means of integral transforms, with the resulting multiple integrals reduced to single finite integrals for the subsonic case. Gakenheimer and Miklowitz [2] derived the transient displacements for the interior of an elastic halfspace under a suddenly applied point load moving at a constant speed along the free surface. All the subsonic, transonic and supersonic cases were studied, while the inverse transform is evaluated by the Carniard-de Hoop technique. The steady-state response for the same problem was also given by Frýba [3] in integral form.

Using a method similar to Eason's [1], Alabi [4] studied the response due to an oblique moving point load applied on the free surface. By numerical integration, a parametric study was performed to investigate the effects of the load speed, distance and ground depth for the subsonic case. De Barrors and Luco [5] obtained the steady-state displace-

\footnotetext{
* Corresponding author. Fax: +886-2-2363-7585.

E-mail address: ybyang@ce.ntu.edu.tw (Y.-B. Yang).
}

ments and stresses within a multi-layered viscoelastic half-space generated by a buried or surface point load moving in a horizontal straight line with subsonic, transonic or supersonic speeds, following generally the procedure proposed by Luco and Aspel [6]. Grundmann et al. [7] studied the response of a layered half-space subjected to a single moving periodic load as well as a simplified train load. The inverse transformation was performed by a decomposition in wavelets [8] and the layered half-space was modeled by one-dimensional finite elements for the vertical direction in the transformed domain.

The objective of this paper is not to give an extensive review of all the related previous works. Rather, efforts are concentrated on investigation of the key parameters involved in wave propagation in a half-space caused by moving vehicles. Four types of vehicle loads are considered, which include a moving point load, a uniformly distributed wheel load, an elastically distributed wheel load, and a train load simulated as a sequence of elastically distributed wheel loads. The elastically distributed wheel load has been considered by Krylov et al. [9,10] and Takemiya [11]. However, they have not proceeded to investigate the effect of vehicles traveling at different speeds.

\section{Governing equations}

The governing equations in terms of the displacements for the homogenous isotropic elastic solid shown in Fig. 1 


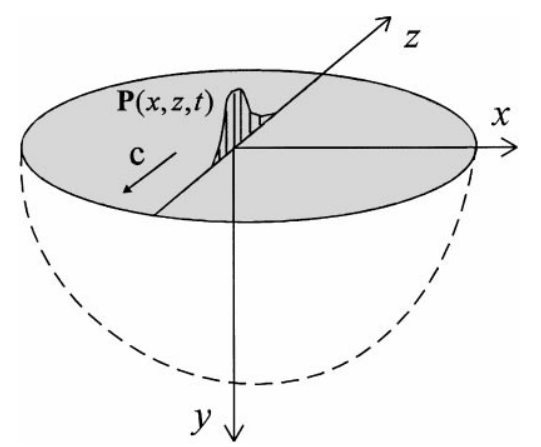

Fig. 1. Uniform visco-elastic half-space subjected to a moving load.

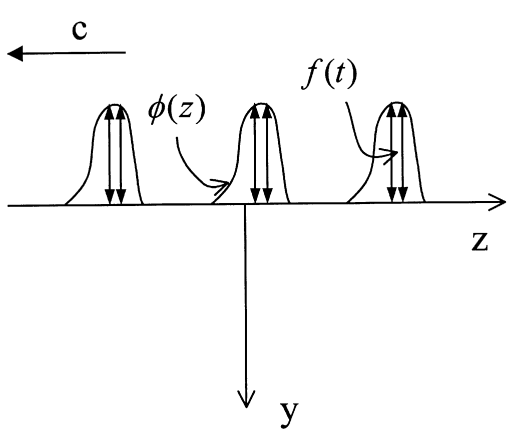

Fig. 2. Schematic of a train-induced general moving load.

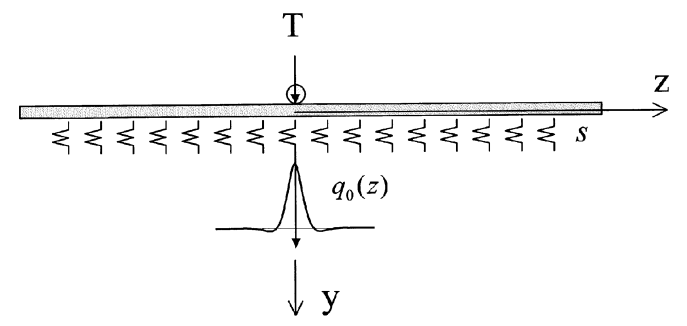

(a)

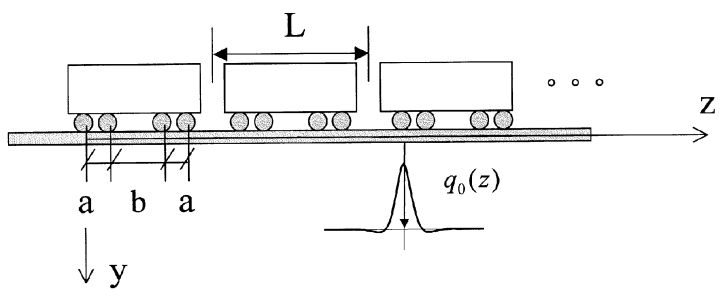

(b)

Fig. 3. Train-induced loadings between rails and soils: (a) single wheel load, (b) a sequence of wheel loads.

can be written as

$$
(\lambda+\mu) \nabla \nabla \cdot \mathbf{u}+\mu \nabla^{2} \mathbf{u}+\rho \mathbf{f}=\rho \ddot{\mathbf{u}}
$$

where $\lambda$ and $\mu$ are Lamé's constants, $\mathbf{u}$ and $\mathbf{f}$ denote the displacement and body force components, respectively, and $\rho$ is the mass density of the elastic solid. The preceding equation can be reduced to a simple set of equations through

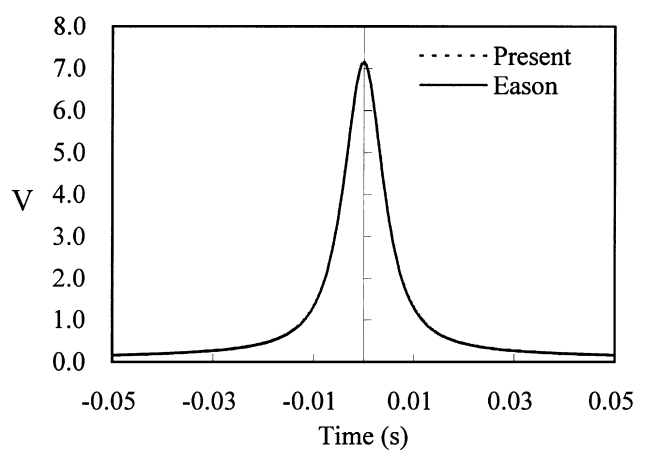

(a)

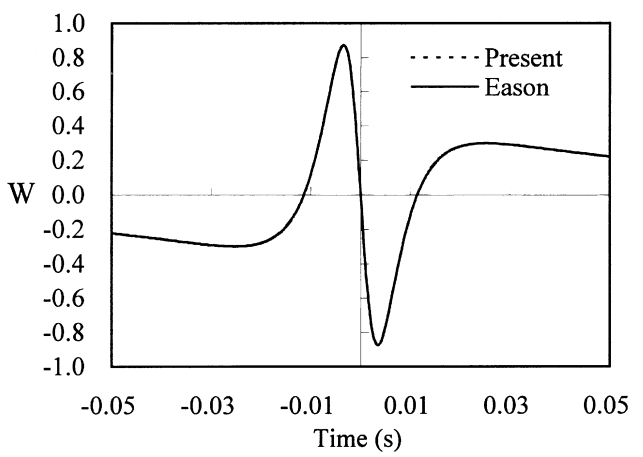

(b)

Fig. 4. Comparison of present results with those of Eason.

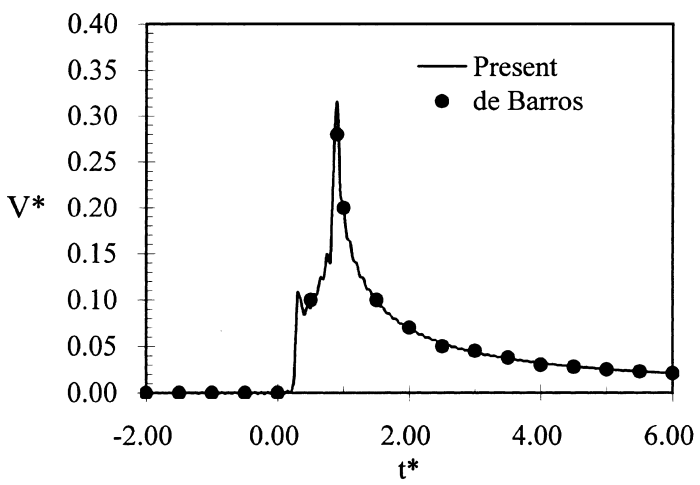

(a)

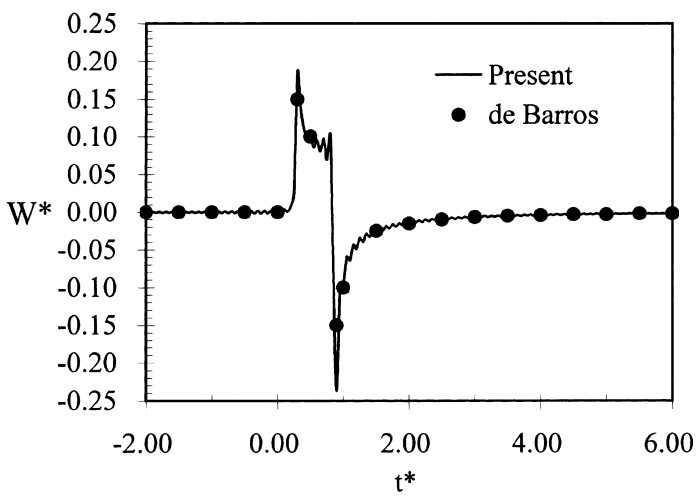

(b)

Fig. 5. Comparison of present results with those of de Barros and Luco. 

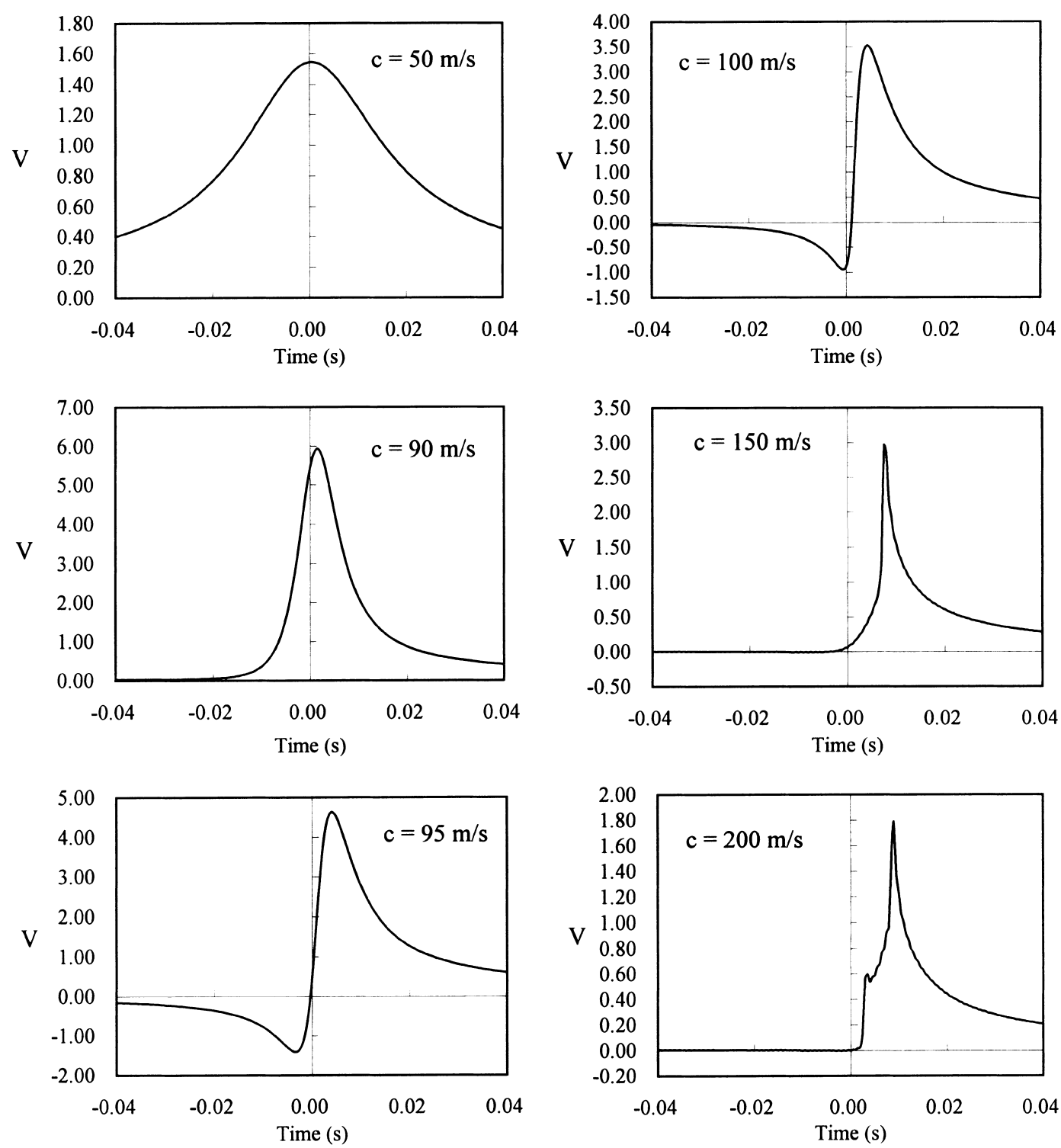

Fig. 6. Vertical displacement $V$ induced by a moving point load.

use of the Helmholtz potential. Let the displacement field $\mathbf{u}$ be represented as

$\mathbf{u}=\nabla \Phi+\nabla \times \mathbf{\Psi}, \quad \Psi_{y}=0$

where $\Phi(\mathbf{x}, t)$ is a scalar function and $\boldsymbol{\Psi}(\mathbf{x}, t)$ a vectorvalued function. The equation $\Psi_{y}=0$ provides a necessary condition for uniquely determining the three components of $\mathbf{u}$ from the four components of $\Phi$ and $\boldsymbol{\Psi}$. In the absence of body forces, one may substitute Eq. (2) into Eq. (1) to obtain

$\Delta \Phi-\frac{1}{c_{\mathrm{P}}^{2}} \frac{\partial^{2} \Phi}{\partial t^{2}}=0, \quad \Delta \boldsymbol{\Psi}-\frac{1}{c_{\mathrm{S}}^{2}} \frac{\partial^{2} \boldsymbol{\Psi}}{\partial t^{2}}=0$

in which the compression and shear wave speeds, $c_{\mathrm{P}}$ and $c_{\mathrm{S}}$, are defined as

$c_{\mathrm{P}}=\sqrt{\frac{\lambda+2 \mu}{\rho}}, \quad c_{\mathrm{S}}=\sqrt{\frac{\mu}{\rho}}$
The first equation with the scalar potential $\Phi$ in Eq. (3) describes the propagation of the compression waves, and the second one with the vectorial potential $\boldsymbol{\Psi}$ the shear waves. The implication from Eq. (3) is that the waves may propagate into the interior of an elastic solid at two different speeds, i.e. at $c_{\mathrm{P}}$ and $c_{\mathrm{S}}$. From Eq. (2), the three components of the displacement $\mathbf{u}$ can be expressed as:

$u=\frac{\partial}{\partial x} \Phi+\frac{\partial}{\partial y} \Psi_{z}$

$v=\frac{\partial}{\partial y} \Phi+\frac{\partial}{\partial z} \Psi_{x}-\frac{\partial}{\partial x} \Psi_{z}$

$w=\frac{\partial}{\partial z} \Phi-\frac{\partial}{\partial y} \Psi_{x}$

where $u, v, w$ are the displacement components in the time 

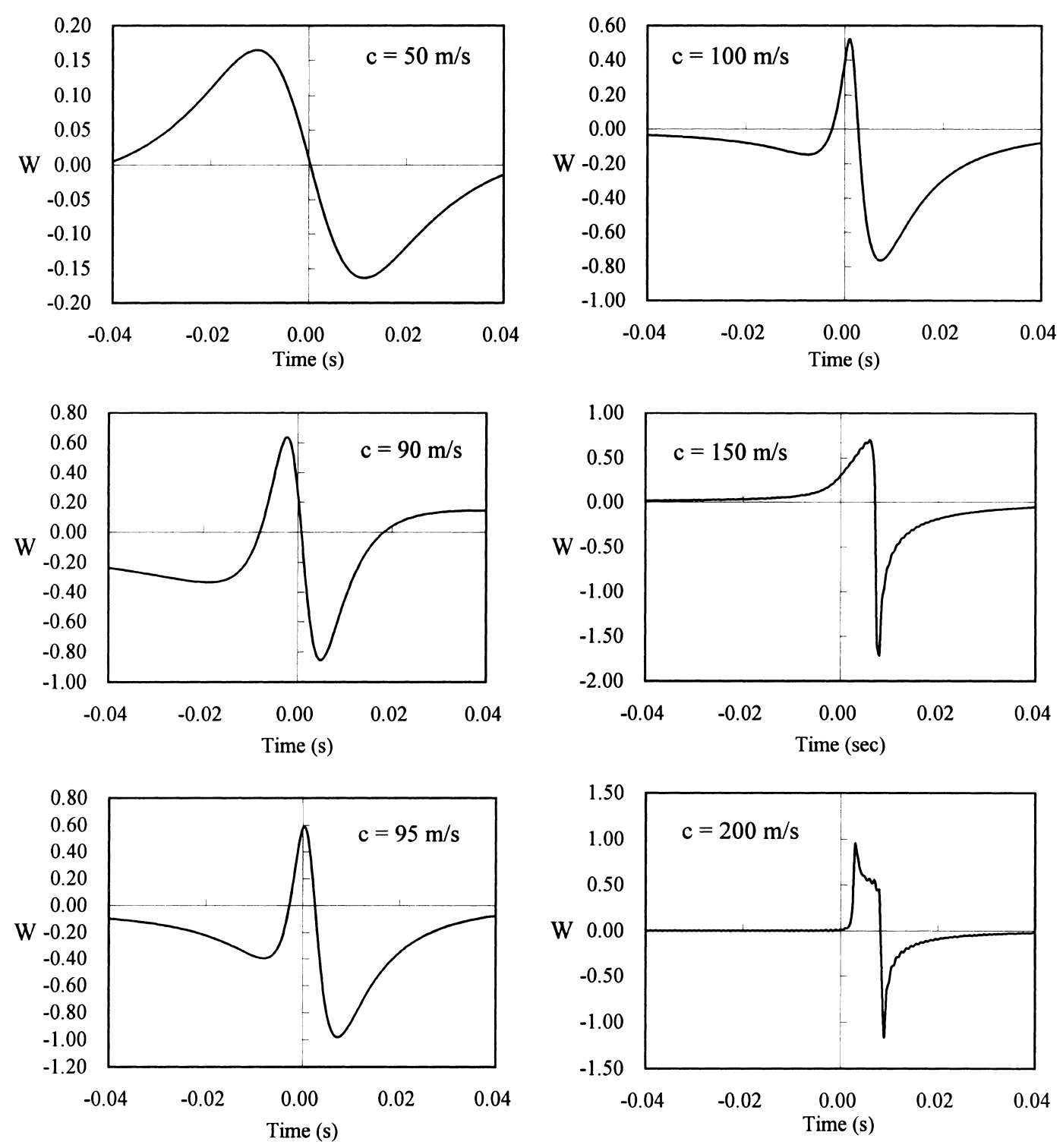

Fig. 7. Longitudinal displacement $W$ induced by a moving point load.

and space domains along the three directions $x, y, z$. By Hooke's law and the strain-displacement relations, the stresses can be expressed in terms of $\Phi$ and $\boldsymbol{\Psi}$ as:

$$
\begin{aligned}
\sigma_{y y} & =2 \mu\left(\frac{\partial^{2}}{\partial y^{2}} \Phi+\frac{\partial^{2}}{\partial y \partial z} \Psi_{x}-\frac{\partial^{2}}{\partial y \partial x} \Psi_{z}\right) \\
& +\lambda\left(\frac{\partial^{2}}{\partial x^{2}}+\frac{\partial^{2}}{\partial y^{2}}+\frac{\partial^{2}}{\partial z^{2}}\right) \Phi \\
\tau_{x y} & =\mu\left[2 \frac{\partial^{2}}{\partial x \partial y} \Phi+\frac{\partial^{2}}{\partial x \partial z} \Psi_{x}+\left(\frac{\partial^{2}}{\partial y^{2}}-\frac{\partial^{2}}{\partial x^{2}}\right) \Psi_{z}\right] \\
\tau_{z y} & =\mu\left[2 \frac{\partial^{2}}{\partial z \partial y} \Phi-\frac{\partial^{2}}{\partial x \partial z} \Psi_{z}-\left(\frac{\partial^{2}}{\partial y^{2}}-\frac{\partial^{2}}{\partial z^{2}}\right) \Psi_{x}\right]
\end{aligned}
$$

for an elastic solid.
In this paper, the triple Fourier transform and its inverse that are adopted throughout are defined as follows:

$$
\begin{aligned}
\hat{f}\left(k_{x}, y, k_{z}, \omega\right)= & \frac{1}{(2 \pi)^{3}} \int_{-\infty}^{\infty} \int_{-\infty}^{\infty} \int_{-\infty}^{\infty} f(x, y, z, t) \\
& \times \exp \left(-i k_{x} x\right) \exp \left(-i k_{z} z\right) \exp (-i \omega t) \mathrm{d} x \mathrm{~d} z \mathrm{~d} t
\end{aligned}
$$

$$
\begin{aligned}
f(x, y, z, t)= & \int_{-\infty}^{\infty} \int_{-\infty}^{\infty} \int_{-\infty}^{\infty} \hat{f}\left(k_{x}, y, k_{z}, \omega\right) \\
& \times \exp \left(i k_{x} x\right) \exp \left(i k_{z} z\right) \exp (i \omega t) \mathrm{d} k_{x} \mathrm{~d} k_{z} \mathrm{~d} \omega
\end{aligned}
$$

Where $k_{x}$ and $k_{z}$ are wave numbers along the $\mathrm{x}$ and $\mathrm{z}$ axes, respectively.

By applying the triple Fourier transformation, one can transform Eq. (3) from partial differential equations into 


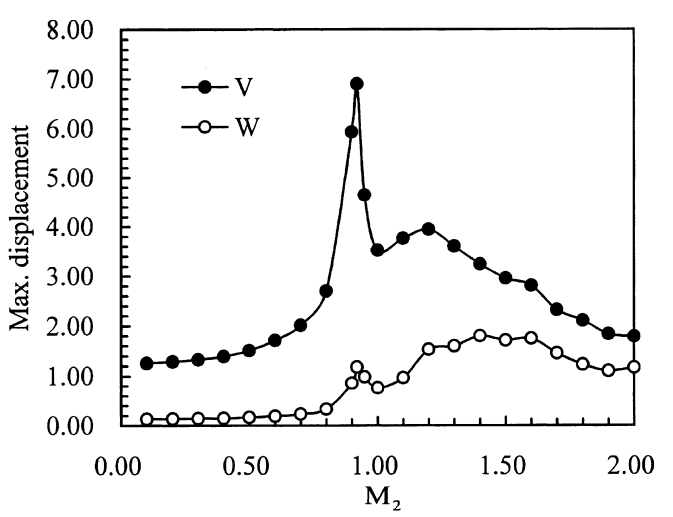

Fig. 8. Maximum displacements induced by the moving point load.

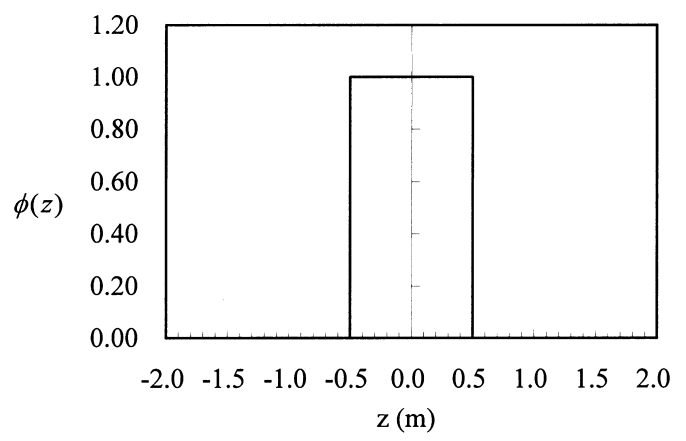

(a)

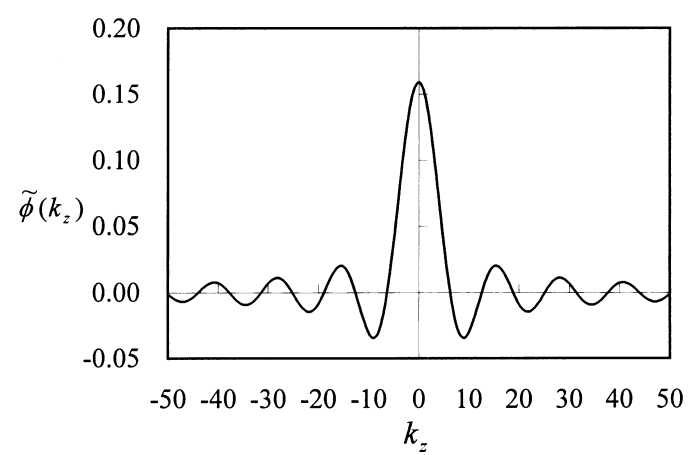

(b)

Fig. 9. A uniformly distributed moving load: (a) load distribution function, (b) Fourier transform.

ordinary differential equations with the vertical coordinate $y$ serving as the variable, that is,

$\left[-k_{x}^{2}-k_{z}^{2}+\left(\frac{\omega}{c_{\mathrm{P}}}\right)^{2}\right] \hat{\Phi}+\frac{\partial^{2}}{\partial y^{2}} \hat{\Phi}=0$,

$$
\left[-k_{x}^{2}-k_{z}^{2}+\left(\frac{\omega}{c_{\mathrm{S}}}\right)^{2}\right] \hat{\mathbf{\Psi}}+\frac{\partial^{2}}{\partial y^{2}} \hat{\mathbf{\Psi}}=0
$$

By letting $k_{\mathrm{P}}=\omega / c_{\mathrm{P}}, k_{\mathrm{S}}=\omega / c_{\mathrm{S}}$ and $m_{1}^{2}=k_{x}^{2}+k_{z}^{2}-k_{\mathrm{P}}^{2}$, $m_{2}^{2}=k_{x}^{2}+k_{z}^{2}-k_{\mathrm{S}}^{2}$, the preceding differential equations

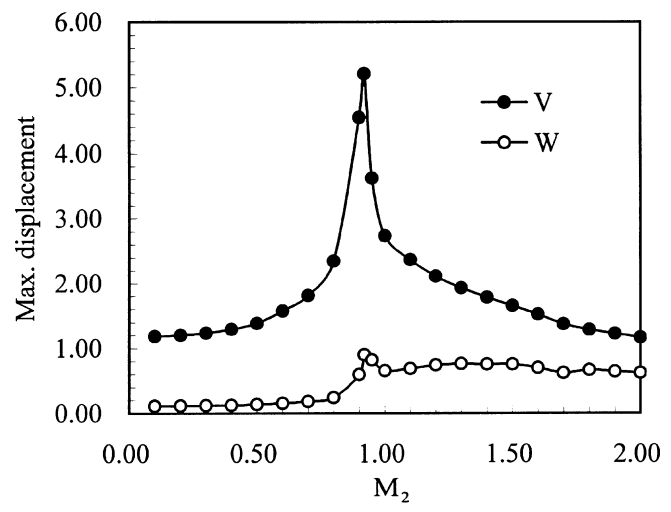

Fig. 10. Maximum displacements induced by a uniformly distributed load.

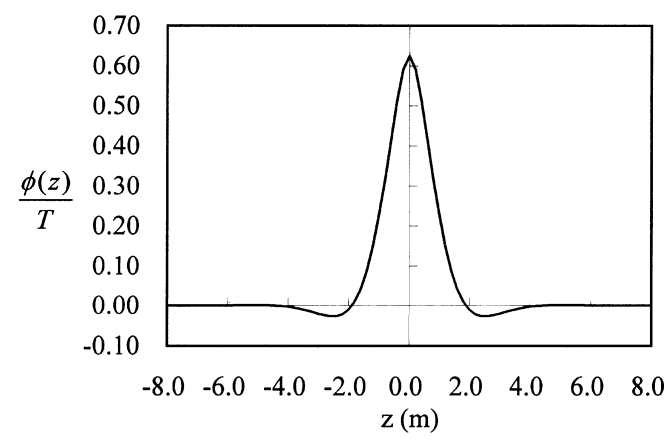

(a)

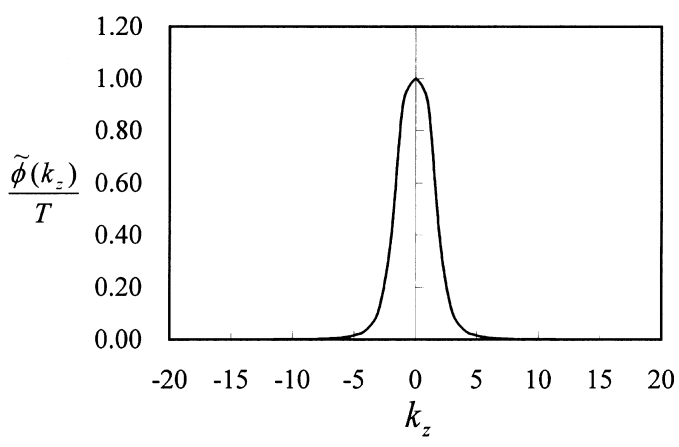

(b)

Fig. 11. An elastically distributed moving load: (a) load distribution function, (b) Fourier transform.

can be rewritten in a more compact form as

$$
\frac{\partial^{2}}{\partial y^{2}} \hat{\boldsymbol{\Phi}}-m_{1}^{2} \hat{\boldsymbol{\Phi}}=0, \quad \frac{\partial^{2}}{\partial y^{2}} \hat{\boldsymbol{\Psi}}-m_{2}^{2} \hat{\boldsymbol{\Psi}}=0
$$

both of which are of the same form.

\section{Solutions for the response}

\subsection{Boundary conditions}

The solution to the transformed differential Eq. (9) can be 
given as

$\hat{\Phi}=A \exp \left(-m_{1} y\right), \quad \hat{\Psi}_{z}=B \exp \left(-m_{2} y\right), \quad \hat{\Psi}_{x}=C \exp \left(-m_{2} y\right)$

where $A, B$ and $C$ are the constants to be determined and the exponentially increasing terms have been discarded because of the radiation and finiteness conditions imposed for the half-space at infinity. Since the solutions to the governing Eq. (9) have been given in the transformed domain, both the displacements and stresses should be expressed in the same domain before the boundary conditions can be imposed. By applying the Fourier transformation to Eqs. (5) and (6), along with substitution of Eq. (10), the transformed displacements and stresses can be expressed in terms of the constants $A, B$ and $C$ as

$$
\begin{aligned}
& \left\{\begin{array}{c}
\hat{u} \\
\hat{v} \\
\hat{w}
\end{array}\right\}=[\mathbf{D}][\mathbf{H}]\left\{\begin{array}{l}
A \\
B \\
C
\end{array}\right\} \\
& \left\{\begin{array}{c}
\hat{\sigma}_{y y} \\
\hat{\tau}_{x y} \\
\hat{\tau}_{z y}
\end{array}\right\}=[\mathbf{S}][\mathbf{H}]\left\{\begin{array}{l}
A \\
B \\
C
\end{array}\right\}
\end{aligned}
$$

where

$$
\begin{aligned}
& {[\mathbf{D}]=\left[\begin{array}{ccc}
i k_{x} & -m_{2} & 0 \\
-m_{1} & -i k_{x} & i k_{z} \\
i k_{z} & 0 & m_{2}
\end{array}\right]} \\
& {[\mathbf{S}]=\left[\begin{array}{ccc}
(2 \mu+\lambda) m_{1}^{2}-\lambda\left(k_{x}^{2}+k_{z}^{2}\right) & 2 i \mu k_{x} m_{2} & -2 i \mu k_{z} m_{2} \\
-2 i \mu k_{x} m_{1} & \mu\left(m_{2}^{2}+k_{x}^{2}\right) & -\mu k_{x} k_{z} \\
-2 i \mu k_{z} m_{1} & \mu k_{x} k_{z} & -\mu\left(m_{2}^{2}+k_{z}^{2}\right)
\end{array}\right]} \\
& {[\mathbf{H}]=\left[\begin{array}{ccc}
e^{-m_{1} y} & 0 & 0 \\
0 & e^{-m_{2} y} & 0 \\
0 & 0 & e^{-m_{2} y}
\end{array}\right]}
\end{aligned}
$$

Consider a uniform elastic half-space subjected to a vertical load $\mathbf{P}(x, z, t)$ moving along the negative $z$ direction as shown in Fig. 1, in which

$\mathbf{P}(x, z, t)=(0, P, 0)$

with $P$ denoting the vertical load component. Accordingly, the boundary conditions on the free surface of the half-space are

$$
\hat{\sigma}_{y y}(y=0)=-\hat{P}, \quad \hat{\tau}_{x y}(y=0)=0, \quad \hat{\tau}_{z y}(y=0)=0
$$

where $\hat{P}$ denotes the applied load in the transformed domain. By substituting Eq. (14) into Eq. (11b), the three constants $A, B$ and $C$ can be solved and expressed in terms of the transformed load. Then, by substituting these constants $A, B, C$ into Eq. (11a), one can obtain the displacements in the transformed domain, also in terms of the transformed load, as

$$
\left\{\begin{array}{c}
\hat{u} \\
\hat{v} \\
\hat{w}
\end{array}\right\}=-[\mathbf{D}][\mathbf{H}][\mathbf{S}]^{-1}\left\{\begin{array}{c}
\hat{P} \\
0 \\
0
\end{array}\right\}
$$

where the inverse of matrix $[\mathbf{S}]$ is

$[\mathbf{S}]^{-1}=\frac{1}{2 \mu Q}[\mathbf{G}]_{3 \times 3}$

and

$Q=\left(k_{x}^{2}+k_{z}^{2}-\frac{1}{2} k_{\mathrm{S}}^{2}\right)^{2}-m_{1} m_{2}\left(k_{x}^{2}+k_{z}^{2}\right)$

The nine entries of the matrix $[\mathbf{G}]$ have been given in the Appendix.

\subsection{Steady state response in time domain}

The final expression of the displacements of the halfspace in time domain can be obtained by employing the inverse Fourier transformation to Eq. (15), that is,

$$
\left\{\begin{array}{l}
u \\
v \\
w
\end{array}\right\}=-\int_{-\infty}^{\infty} \int_{-\infty}^{\infty} \int_{-\infty}^{\infty}[\mathbf{D}][\mathbf{H}]
$$

$$
\times[\mathbf{S}]^{-1}\left\{\begin{array}{c}
\hat{P} \\
0 \\
0
\end{array}\right\} \exp \left(i k_{x} x\right) \exp \left(i k_{z} z\right) \exp (i \omega t) \mathrm{d} k_{x} \mathrm{~d} k_{z} \mathrm{~d} \omega
$$

Similarly, the velocities and accelerations in time domain can be written as:

$$
\begin{aligned}
\left\{\begin{array}{c}
\dot{u} \\
\dot{v} \\
\dot{w}
\end{array}\right\}= & -\int_{-\infty}^{\infty} \int_{-\infty}^{\infty} \int_{-\infty}^{\infty} i \omega[\mathbf{D}][\mathbf{H}] \\
& \times[\mathbf{S}]^{-1}\left\{\begin{array}{c}
\hat{P} \\
0 \\
0
\end{array}\right\} \exp \left(i k_{x} x\right) \exp \left(i k_{z} z\right) \exp (i \omega t) \mathrm{d} k_{x} \mathrm{~d} k_{z} \mathrm{~d} \omega
\end{aligned}
$$

$$
\begin{aligned}
\left\{\begin{array}{c}
\ddot{u} \\
\ddot{v} \\
\ddot{w}
\end{array}\right\}= & \int_{-\infty}^{\infty} \int_{-\infty}^{\infty} \int_{-\infty}^{\infty} \omega^{2}[\mathbf{D}][\mathbf{H}] \\
& \times[\mathbf{S}]^{-1}\left\{\begin{array}{c}
\hat{P} \\
0 \\
0
\end{array}\right\} \exp \left(i k_{x} x\right) \exp \left(i k_{z} z\right) \exp (i \omega t) \mathrm{d} k_{x} \mathrm{~d} k_{z} \mathrm{~d} \omega
\end{aligned}
$$

which are all functions of the load component $\hat{P}$. 

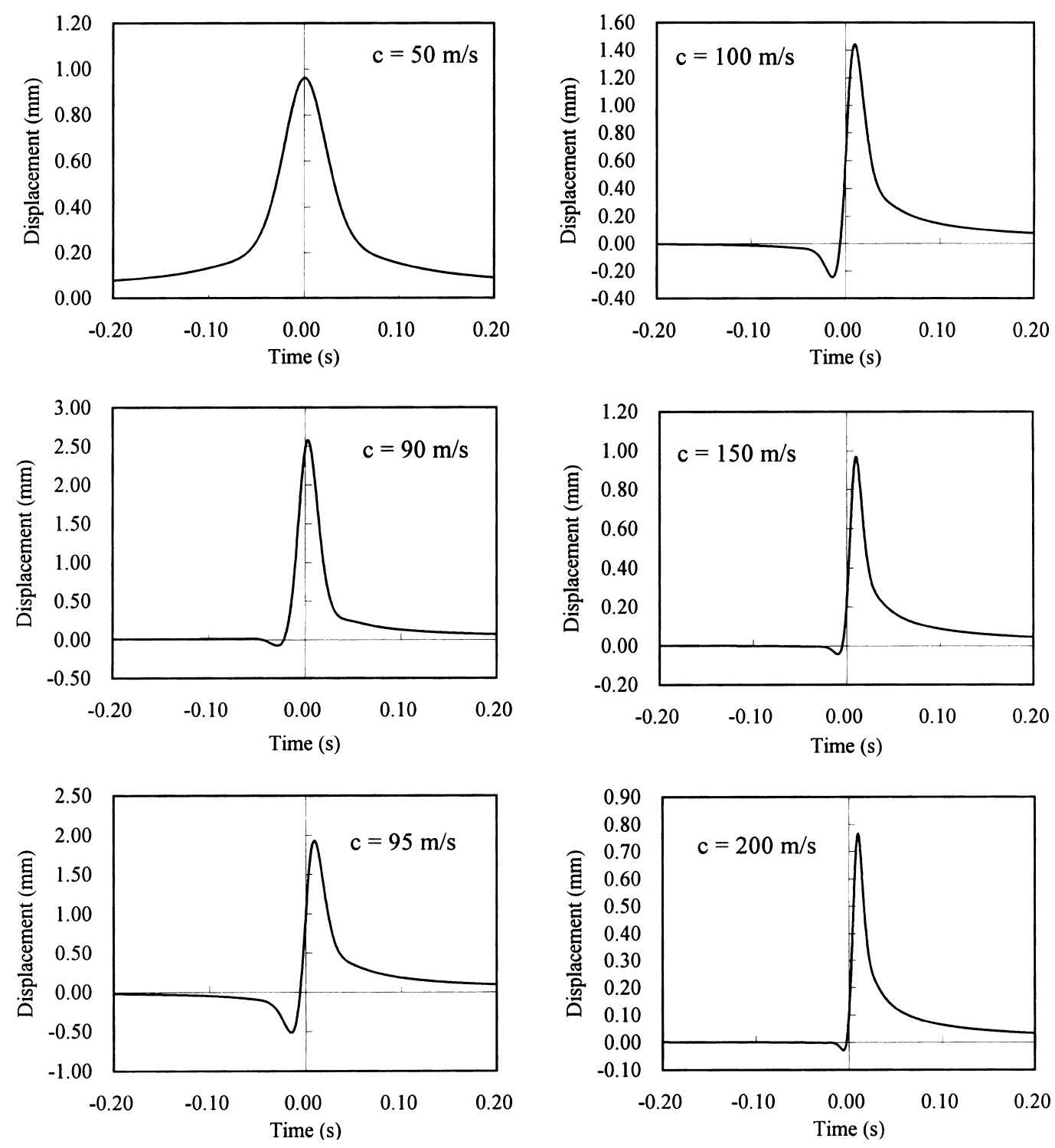

Fig. 12. Vertical displacement for an elastically distributed load $\left(f_{0}=0 \mathrm{~Hz}\right)$.

\section{Loading functions for moving loads of different forms}

\subsection{General loading function of a moving train}

Train-induced vibrations are caused in soils by loadings transmitted to the track and underlying soils through the contact points existing between the wheels and rails. In general, the loadings can be split up into two parts as depicted in Fig. 2. The first part relates to the distribution of the axle loads passing a fixed point given as $\phi(z-c t)$, where $c$ is the speed of the moving vehicles and $\phi(z)$ the distribution pattern of the axle loads. This part may lead to excitation at the vehicles passing frequencies and is the major source for the train speed-dependent components of the low frequency vibration spectra [10]. The second part is generated by the interaction between the wheels and rails, which, as indicated by $f(t)$, is moving with the wheels and independent of the moving direction $z$. For the present case, the moving load $\mathbf{P}(x, z, t)$ can be expressed as

$P=\delta(x) \phi(z-c t) f(t)$

Both the functions $\phi(z)$ and $f(t)$ will be determined in the following sections. By applying the Fourier transformation to Eq. (21), the moving load can be expressed as

$\hat{P}=\frac{1}{2 \pi} \tilde{\phi}\left(k_{z}\right) \tilde{f}\left(\omega+k_{z} c\right)$

in which $\tilde{\phi}\left(k_{z}\right)$ and $\tilde{f}(\omega)$ are the one-dimensional Fourier transforms of $\phi(z)$ and $f(t)$, respectively, with respect to $z$ and $t$. In this paper, the symbols $\hat{.}$ and $\tilde{.}$ are used to denote a triple and one-dimensional Fourier transform, respectively. 

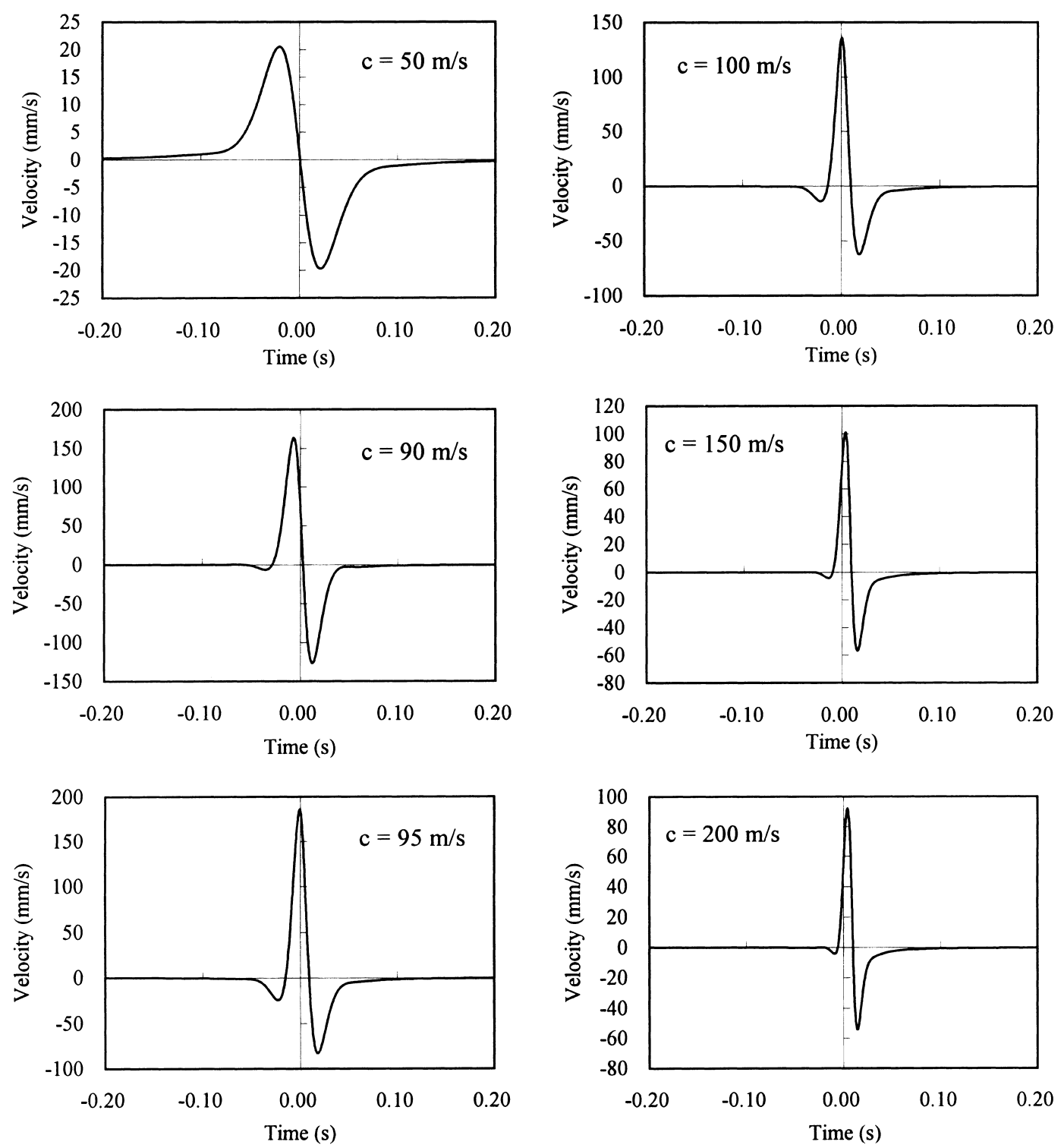

Fig. 13. Vertical velocity for an elastically distributed load $\left(f_{0}=0 \mathrm{~Hz}\right)$.

\subsection{Distribution function $\phi(z)$ of the loading}

Theoretically, the distribution function should be determined based on field data collected for the wheel loads. However, using simple models enables us to grasp the most fundamental features of vehicle-induced ground vibrations. Moreover, most related previous works that can be used as references deal only with the case of moving point loads. Based on these considerations, four different forms of distribution function $\phi(z)$, ranging from a single point load to a rather sophisticated train load with multi wheels, will be considered, along with their Fourier transforms $\tilde{\phi}\left(k_{z}\right)$ given in analytical form.

\subsubsection{Single moving point load}

For a point load, the distribution function along the $z$-axis can be written in the form of Dirac's delta function as:

$\phi(z)=\delta(z)$

Correspondingly, the Fourier transform is

$\tilde{\phi}\left(k_{z}\right)=\frac{1}{2 \pi}$

which is a constant regardless of the value of $k_{z}$.

\subsubsection{A uniformly distributed wheel load}

In reality, the contact point existing between a wheel and rail is not actually a "point" but an "area". Thus, a better representation for the wheel load is a uniformly distributed load given as:

$\phi(z)=\left\{\begin{array}{cl}1 /(2 a) & -a \leq z \leq a \\ 0 & \text { otherwise }\end{array}\right.$ 

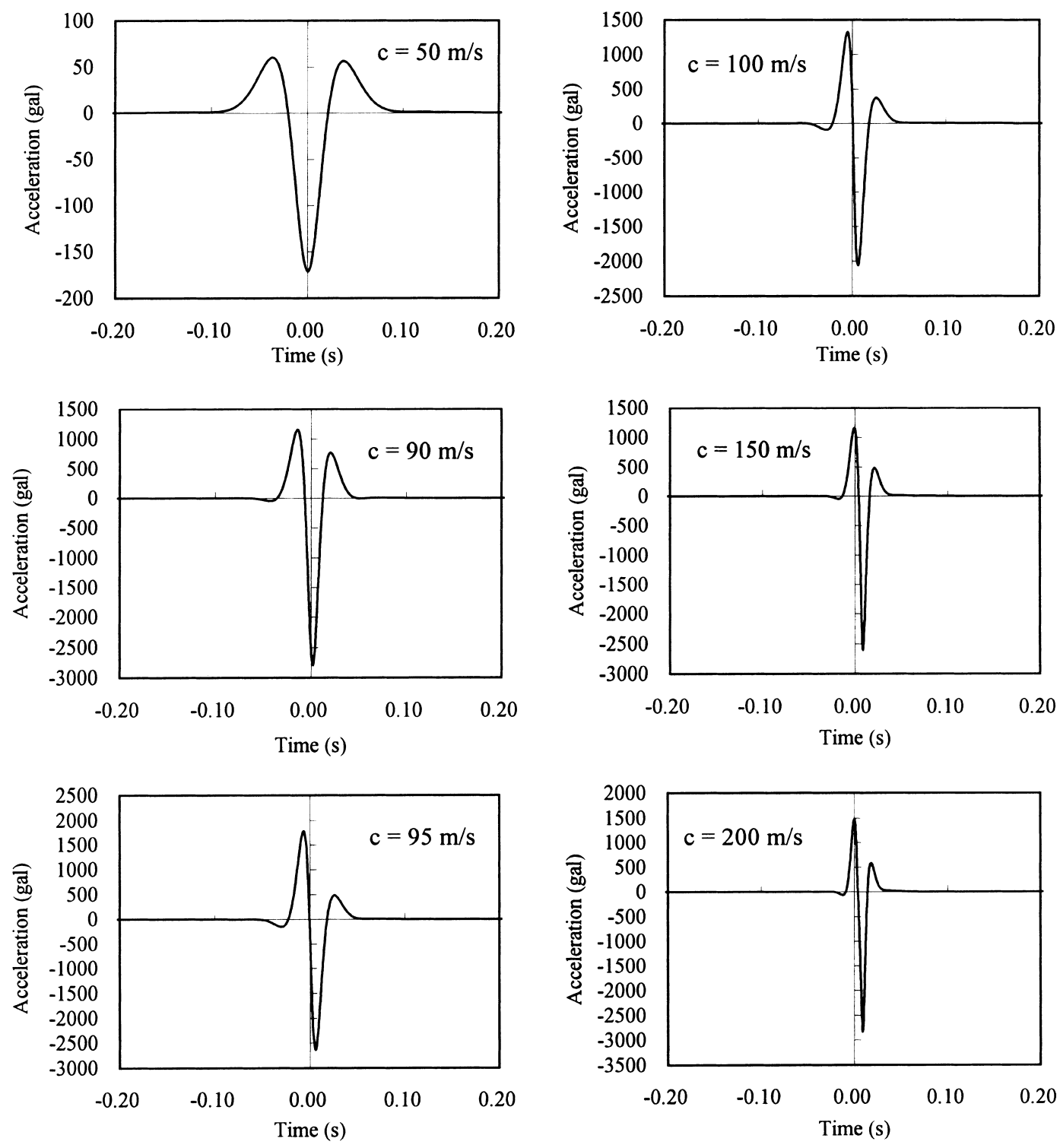

Fig. 14. Vertical acceleration for an elastically distributed load $\left(f_{0}=0 \mathrm{~Hz}\right)$.

where $a$ is a constant, representing the half width of the distributed load. Based on the definition of $\phi(z)$ given in Eq. (25), the integration of $\phi(z)$ from $-\infty$ to $\infty$ is 1 , implying that the total contribution of the loading function equals a unit value, same as that implied by Dirac's delta function. By applying the Fourier transformation to Eq. (25), the transformed load $\tilde{\phi}\left(k_{z}\right)$ can be obtained as

$\tilde{\phi}\left(k_{z}\right)=\left\{\begin{array}{c}\frac{\sin \left(a k_{z}\right)}{\pi k_{z}} \quad k_{z} \neq 0 \\ \frac{a}{\pi} \quad k_{z}=0\end{array}\right.$

As can be seen, the transformed function $\tilde{\phi}\left(k_{z}\right)$ is no longer a constant, but tends to decay with the increase of $k_{z}$.

\subsubsection{An elastically distributed wheel load}

If the wheel load is regarded as the force exerted from the track onto the underlying soils, rather than the one from the wheels onto the track, one may use the deflection curve of the track to simulate the distribution of the wheel load [911]. In this connection, the track is treated as an infinite Bernoulli-Euler beam supported by an elastic foundation of stiffness $s$ as shown in Fig. 3(a). For an elastically supported beam with an axle load $T$ acting at $z=0$, the vertical displacement $v$ is [12]:

$v(z)=\frac{T}{2 s \alpha} \exp \left(\frac{-|z|}{\alpha}\right)\left[\cos \left(\frac{|z|}{\alpha}\right)+\sin \left(\frac{|z|}{\alpha}\right)\right]$

where the characteristic length $\alpha$ can be related to the bending stiffness $E I$ as

$\alpha=\sqrt[4]{\frac{4 E I}{s}}[\mathrm{~m}]$

Consequently, the distribution loading function can be 

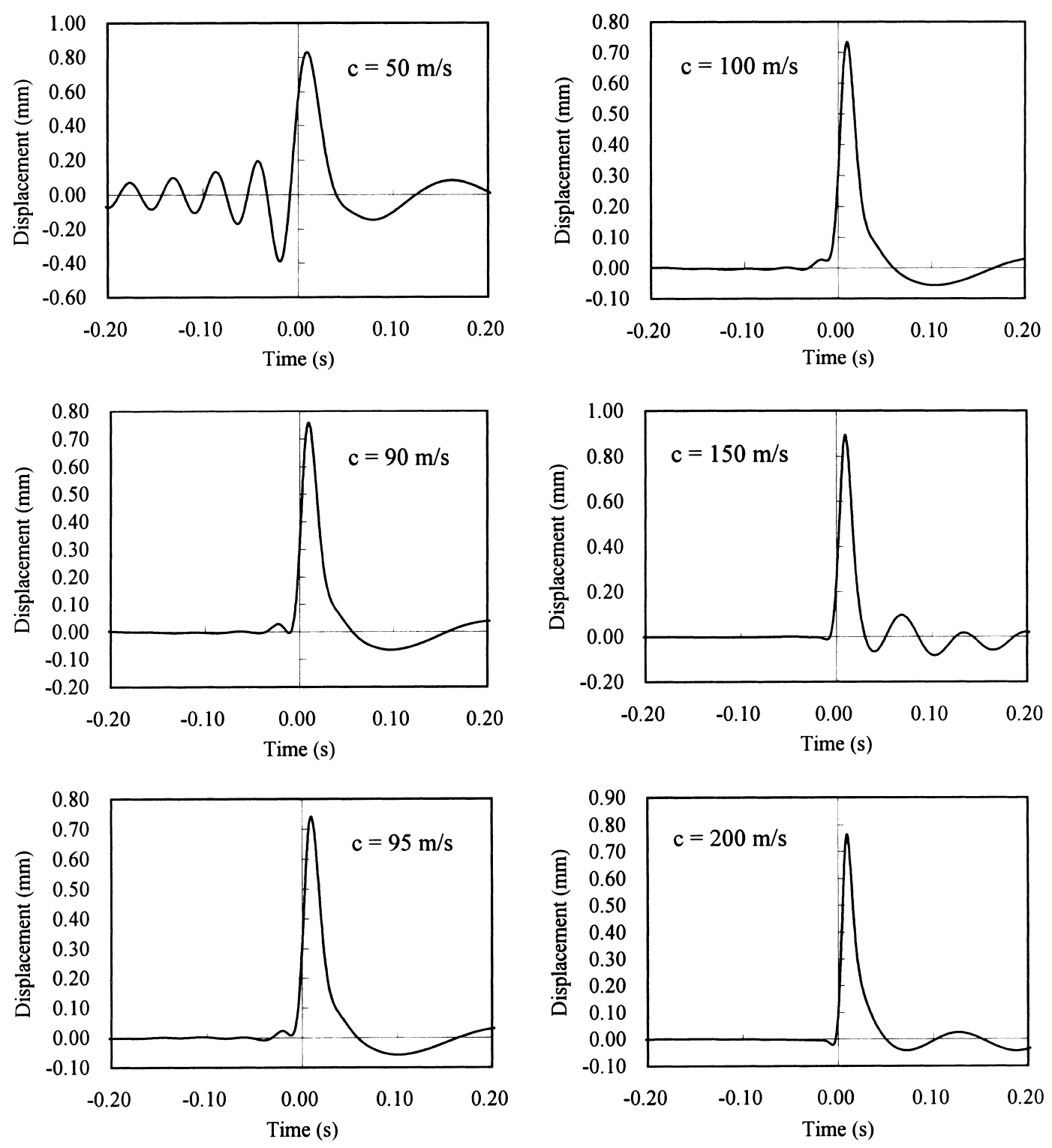

Fig. 15. Real-part vertical displacement for an elastically distributed load $\left(f_{0}=10 \mathrm{~Hz}\right)$.

written as

$\phi(z)=q_{0}(z)=\frac{T}{2 \alpha} \exp \left(\frac{-|z|}{\alpha}\right)\left[\cos \left(\frac{|z| \mid}{\alpha}\right)+\sin \left(\frac{|z|}{\alpha}\right)\right]$

Note that the integration of $\phi(z)$ from $z=-\infty$ to $\infty$ equals the axle load $T$. Obviously, the total force transmitted through the rails remains unchanged, although the pattern of distribution has been changed. By applying the Fourier transformation to Eq. (29), the distribution function can be transformed as

$\tilde{\phi}\left(k_{z}\right)=\tilde{q}_{0}\left(k_{z}\right)=\frac{4 T}{4+k_{z}^{4} \alpha^{4}}$

which is a function of the characteristic length $\alpha$.

\subsubsection{A sequence of moving wheel loads}

Let us extend the single wheel load case to the case of a train consisting of $N$ carriages of equal length $L$ as depicted in Fig. 3(b), where each carriage has two bogies separated by distance $b$, each of which in turn comprises two axles, i.e. two sets of wheels, separated by distance $a$. Suppose that each set of wheels has the same loading distribution function $q_{0}(z)$ as the one given in Eq. (29). The total distribution function of loading for the present case can be written as:

$\phi(z)=\sum_{n=0}^{N-1}\left[q_{0}(z-n L)+q_{0}(z-n L-a)+q_{0}(z-n L-a\right.$

$\left.-b)+q_{0}(z-n L-2 a-b)\right]$ 


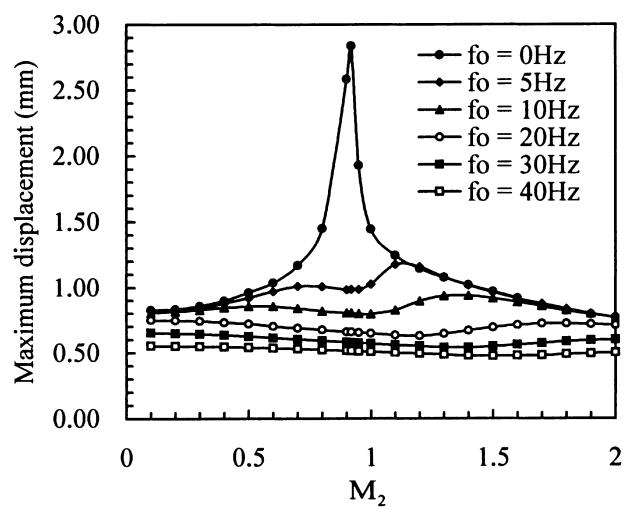

(a)

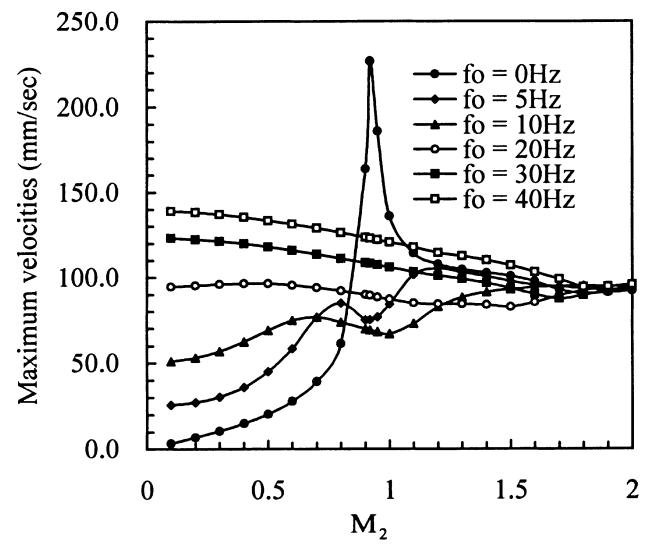

(b)

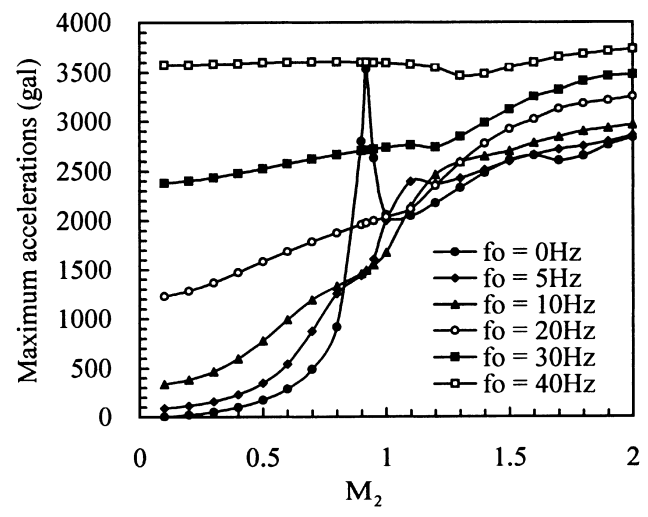

(c)

Fig. 16. Maximum responses induced by an elastically distributed load: (a) displacement, (b) velocity, (c) acceleration.

along with its Fourier transform as

$$
\begin{aligned}
\tilde{\phi}\left(k_{z}\right)= & \tilde{q}_{0}\left(k_{z}\right) \sum_{n=0}^{N-1} \exp \left(-i k_{z} n L\right)\left\{1+\exp \left(-i k_{z} a\right)+\exp [\right. \\
& \left.\left.-i k_{z}(a+b)\right]+\exp \left[-i k_{z}(2 a+b)\right]\right\}
\end{aligned}
$$

where $\tilde{q}_{0}\left(k_{z}\right)$ is the same as the one given in Eq. (30).

\subsection{Interaction forces between wheels and rails}

In general, the interaction force $f(t)$ between the wheels and rails may be simulated by a quasi-static term of constant value plus a dynamic term that varies with time $t$ [12]. The static term is contributed mainly by the wheel weight, whereas the dynamic term by the track irregularities and vehicle defects, such as wheel flats, natural vibrations and hunting. The dynamic term is extremely complex and is by no means generally accessible, due to the fact that the train always interacts with the track, and in turn, with the soils, during its movement. Unless all the carriages of a train, together with track and underground soils, are accurately included in an analytical model, the actual interaction force can never be found. In the present study, the dynamic term $f(t)$ is assumed to depend on only a single frequency $\omega_{0}$, i.e. $f(t)=\exp \left(i \omega_{0} t\right)$. By so setting, it is easy to see that as $\omega_{0}=0, f(t)=1$, implying that a moving load with no oscillation is considered. For the case with $\omega_{o} \neq 0$, the moving load oscillates by itself at a constant frequency $f_{0}=\omega_{0} /(2 \pi) \mathrm{Hz}$. The following are the ranges of frequencies that may be induced by a moving train: (a) sprung mass: 0-20 Hz; (b) unsprung mass: 0-125 Hz; (c) corrugations, welds, and wheel flats: $0-2000 \mathrm{~Hz}$ [12].

By setting $f(t)=\exp \left(i \omega_{0} t\right)$, the dynamic function $\tilde{f}(\omega+$ $k_{z} c$ ) in Eq. (22) can be expressed as

$\tilde{f}\left(\omega+k_{z} c\right)=\frac{1}{c} \delta\left(\frac{\omega-\omega_{0}}{c}+k_{z}\right)$

which has been given in analytical form.

\subsection{Calculation of inverse Fourier transform}

At this stage, both the functions $\tilde{\phi}\left(k_{z}\right)$ and $\tilde{f}\left(\omega+k_{z} c\right)$ have been made available. By substituting these two functions into Eq. (22) and, in turn, into Eqs. (18)-(20), the final responses of the soils in time domain can be obtained. Note that the inversion of the Fourier transform with respect to $z$ in Eqs. (18)-(20) can be done analytically by only replacing $k_{z}$ with $-\left(\omega-\omega_{0}\right) / c$ because of the involvement of Dirac's delta function in Eq. (33). As a result, the original triple integral is reduced to a double integral with respect to frequency $\omega$ and wave number $k_{x}$ only.

By carefully examining Eqs. (18)-(20), we find that there exist two radicals (as represented by $m_{1}=0$ and $m_{2}=0$ ) and a singularity (as represented by $Q=0$ ) in the integrands. This makes the evaluation of these integrals a formidable task. Because of this, equations similar to Eqs. (18)-(20) were frequently presented in integral form in most previous works. However, over the last decade, more and more researchers tend to evaluate integrals of this sort by numerical methods that can automatically skip the singularities and yield the desired results. In this study, appropriate quadrature routines available in IMSL [13] will be used to perform the inverse transform with respect to $k_{x}$ and the fast Fourier transform with respect to $\omega$. For the cases of 

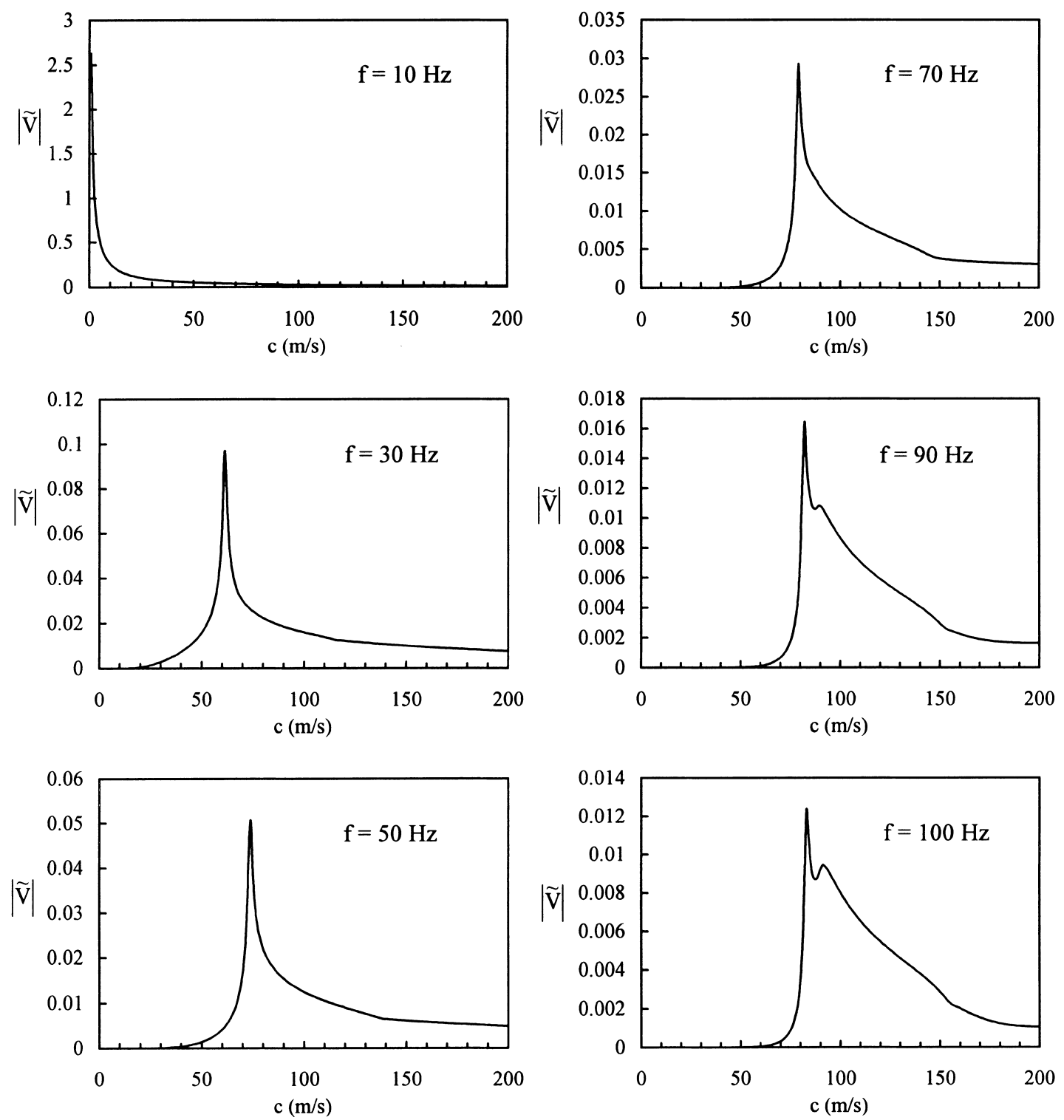

Fig. 17. Displacement in frequency domain for a moving point load $\left(f_{0}=10 \mathrm{~Hz}\right)$.

transonic and supersonic speeds, the pole of the integrands can be shifted off the real axis $k_{x}$ by introducing material attenuation. The material damping can be taken into account through use of complex Lamé's constants, i.e. $\mu^{*}=\mu(1+$ $2 i \beta)$ and $\lambda^{*}=\lambda(1+2 i \beta)$, where $\beta$ denotes the hysteretic damping ratio.

\section{Numerical studies and discussions}

\subsection{Verification of present approach}

The moving point load was the model frequently adopted by researchers in their study of vehicle-induced vibrations. For the purpose of verification, we shall adopt here the same model to study the response of an elastic half-space, assuming the load to move at a subsonic speed. The steady-state response for the same problem was presented by Eason [1] by reducing the resulting multiple integrals to single finite integrals. The Eason results used as the reference herein have been obtained by using subroutines available in IMSL for evaluating these single finite integrals.

For this example, the load is assumed to move at $c=90 \mathrm{~m} / \mathrm{s}$. The elastic half-space considered has a shear wave (S-wave) speed of $c_{\mathrm{S}}=100 \mathrm{~m} / \mathrm{s}$, Poisson's ratio $v=$ 0.25 and mass density $\rho=2000 \mathrm{~kg} / \mathrm{m}^{3}$. The corresponding compression wave (P-wave) speed $c_{\mathrm{P}}$ and Rayleigh wave (R-wave) speed $c_{\mathrm{R}}$ are 173.2 and $92 \mathrm{~m} / \mathrm{s}$, respectively. In Fig. 4, the normalized displacements $V$ and $W$ computed for the observation point $(x, y, z)=\left(0, y_{0}, 0\right)$, with $y_{0}$ set to be $1 \mathrm{~m}$, have been plotted against time $t$, where $V=$ $2 \pi \mu y_{0} \nu / P$ and $W=2 \pi \mu y_{0} w / P$. As can be seen, the results obtained by the present approach are in good agreement with those based on Eason [1]. 


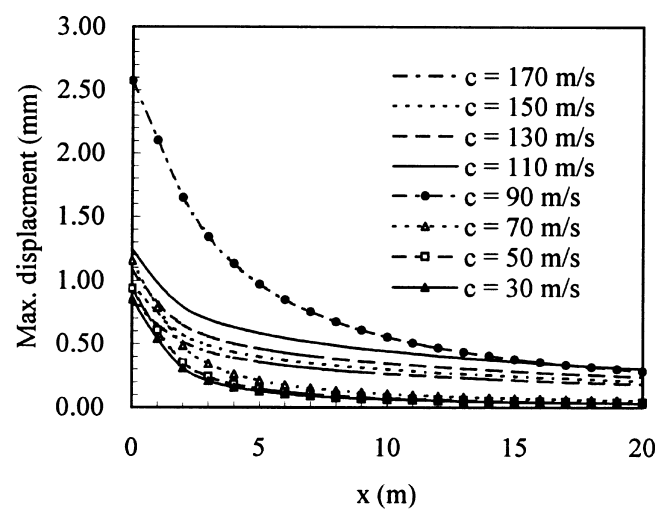

(a)

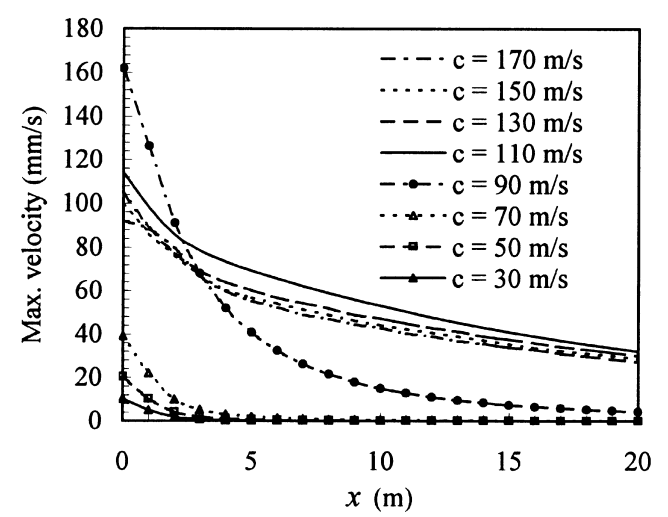

(b)

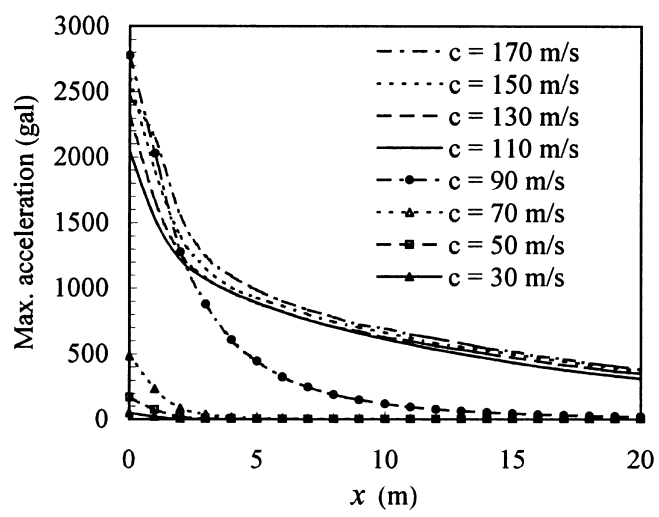

(c)

Fig. 18. Response attenuation for an elastically distributed moving load: (a) displacement, (b) velocity, (c) acceleration.

To verify the accuracy of the present procedure on application to the supersonic range, the displacements $V^{*}$ and $W^{*}$ at the observation point $(0,1 \mathrm{~m}, 0)$ are computed for a point load moving at speed $c=200 \mathrm{~m} / \mathrm{s}$ against the normalized time $t^{*}$ in Fig. 5, where $V^{*}=\mu y_{0} v / P, W^{*}=\mu y_{0} w / P$, and $t^{*}=c_{\mathrm{S}} t / y_{0}$. The solid considered has the same properties as the preceding case, except that a material damping of $\beta=0.01$ is considered for the present case. As can be seen, the present results are in good agreement with those of de Barros and Luco [5].

In the preceding two examples, the present approach has

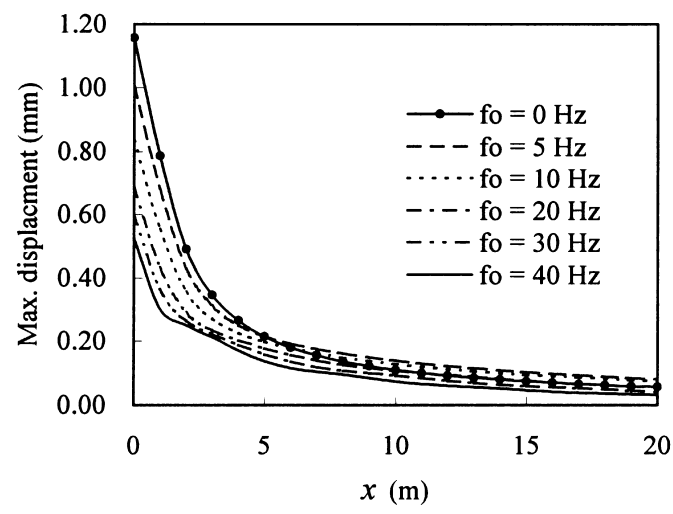

(a)

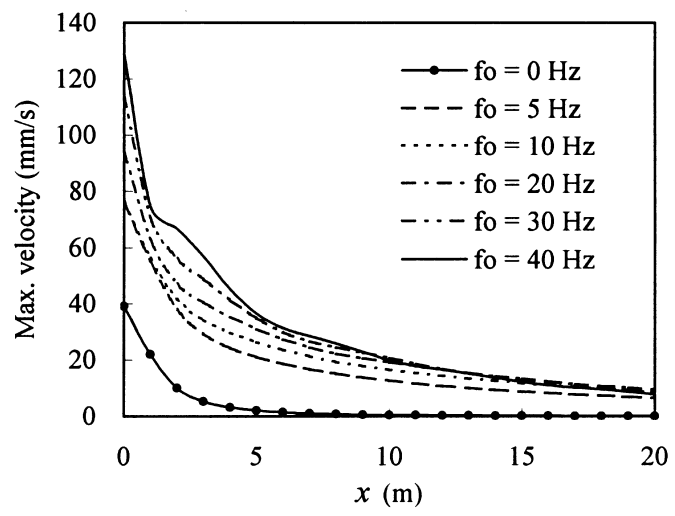

(b)

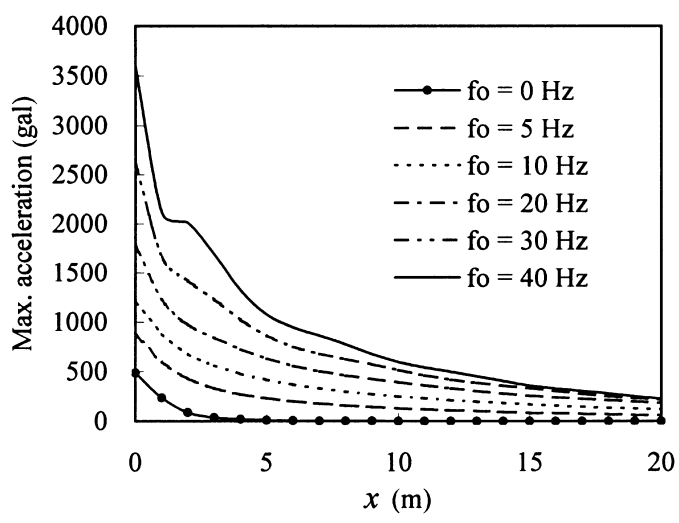

(c)

Fig. 19. Response attenuation for an elastically distributed load moving at $c=70 \mathrm{~m} / \mathrm{s}$ with different frequencies $f_{0}$ : (a) displacement, (b) velocity, (c) acceleration.

been demonstrated to be capable of computing the soil displacements for both the subsonic and supersonic cases. In what follows, the same procedure will be adopted to study the influence of moving speeds for different load distribution functions. Throughout the following analyses, the visco-elastic half-space considered is assumed to have an S-wave speed $c_{\mathrm{S}}=100 \mathrm{~m} / \mathrm{s}, \quad$ P-wave speed $c_{\mathrm{P}}=173.2 \mathrm{~m} / \mathrm{s}, \quad$ R-wave speed $c_{\mathrm{R}}=92 \mathrm{~m} / \mathrm{s}$, Poisson's ratio $v=0.25$, mass density $\rho=2000 \mathrm{~kg} / \mathrm{m}^{3}$, and damping ratio $\beta=0.02$. 


\subsection{Single moving point load}

To evaluate the effect of load moving speeds, the normalized displacements $V$ and $W$, respectively computed at the observation point $(0,1 \mathrm{~m}, 0)$ for the solid subjected to loads moving at the subsonic $(c<100 \mathrm{~m} / \mathrm{s})$, transonic $(100 \mathrm{~m} /$ $\mathrm{s}<c<173.2 \mathrm{~m} / \mathrm{s})$ and supersonic $(c>173.2 \mathrm{~m} / \mathrm{s})$ ranges in Figs. 6 and 7, where the instant $t=0$ corresponds to the moment at which the point load passes through the origin $(z=0)$. As can be seen, the shapes of the responses for load moving speeds in the subsonic region $(c<100 \mathrm{~m} / \mathrm{s})$ are generally different, depending on whether the speed is lower or greater than the R-wave speed $c_{\mathrm{R}}=92 \mathrm{~m} / \mathrm{s}$.

The response is almost symmetric for $V$ or anti-symmetric for $W$ with respect to $t=0$ for load moving speeds lower than the R-wave speed. For the case of zero damping, it becomes entirely symmetric or anti-symmetric, as shown in Fig. 4. As the speed increases and becomes larger than the R-wave speed, the response becomes more asymmetrical as a result of the Mach radiation effect. For the transonic case $(100 \mathrm{~m} / \mathrm{s}<c<173.2 \mathrm{~m} / \mathrm{s})$, the first peak of the response occurring after the instant $t=0$ corresponds to the arrival of the S-wave front, followed immediately by the R-wave front. However, the $\mathrm{S}$ - and $\mathrm{R}$-wave fronts are so close to each other that they cannot be clearly distinguished. For the supersonic case $(c=200 \mathrm{~m} / \mathrm{s})$, the first peak of the response immediately after the instant $t=0$ represents the arrival of the $\mathrm{P}$-wave front, followed by the arrival of the S- and R-wave fronts, which are very close to each other.

The maximum displacements computed at the observation point $(0,1 \mathrm{~m}, 0)$ have been plotted with respect to the $S$ wave Mach number defined as $M_{2}=c / c_{\mathrm{S}}$ in Fig. 8. Here, the range $M_{2}<1.0$ should be interpreted as the subsonic range, $1.0<M_{2}<1.73$ the transonic range, and $M_{2}>1.73$ the supersonic range. As can be seen, the critical speed is equal to the R-wave speed, as indicated by $M_{2}=0.92$. The general trend for the displacements is that as the moving speed increases, they all show a tendency to increase until the critical (resonant) speed is reached, and then they all decrease. However, the vertical displacement $V$ increases at a rate faster than the longitudinal displacement $W$ before the critical speed is reached. Besides, the former attains its maximum at the first critical speed $\left(c=c_{\mathrm{R}}\right)$, while the latter at a speed higher than the R-wave speed $c_{\mathrm{R}}$.

As can be verified from Fig. 8, for the special case of zero moving speed, the present problem reduces to that of a static point force acting at the origin, known as the classical Boussinesq's problem. According to Fung [14], the vertical displacement $v$ for a Boussinesq's problem with load $P$ is

$v=\frac{P}{4 \pi \mu R}\left[2(1-v)+\frac{y^{2}}{R^{2}}\right], \quad R^{2}=x^{2}+y^{2}+z^{2}$

which yields a value of 1.25 for the normalized displa- cement at the observation point for the case with $v=0.25$, exactly the same as the one shown in the figure for $M_{2}=0$.

\subsection{A Uniformly distributed moving wheel load}

Consider a uniformly distributed moving wheel load with $a=0.5$ (see Eq. (25) for definition). For the present case, the load distribution function $\phi(z)$ and its Fourier transform $\tilde{\phi}\left(k_{z}\right)$ have been drawn in Fig. 9(a) and (b). The maximum displacements computed for the point $(0,1 \mathrm{~m}, 0)$ with respect to different $S$-wave Mach numbers $M_{2}$ have been plotted in Fig. 10, from which a trend similar to that of Fig. 8 can be observed, except that the response amplitudes are much lower throughout all the speed range. Such a result is consistent with the understanding that for the same load to be distributed in a wider area, the response induced should be smaller.

Besides, the maximum displacements in Fig. 10 show a trend much smoother than that of Fig. 8. One reason for this is that the contribution of $\tilde{\phi}\left(k_{z}\right)$ for a uniformly distributed load is mostly concentrated on the lower $k_{z}$, as can be seen from Fig. 9(b), thus only a small range of frequencies need be considered when performing the inverse FFT. For the case of a point load, however, the function $\tilde{\phi}\left(k_{z}\right)$ is a constant, as indicated by Eq. (24), implying that "all" frequencies should be considered to guarantee convergence of the maximum displacements to the exact ones. In this study, the range of frequencies considered is $0-1000 \mathrm{~Hz}$.

\subsection{An elastically distributed moving wheel load}

Consider an elastically distributed moving wheel load as defined in Eq. (29), assuming the wheel load $T=10 \mathrm{t}$ and the characteristic length $\alpha=0.8 \mathrm{~m}$. The load distribution function and its transform were shown in Fig. 11(a) and (b). The vertical displacements, velocities and accelerations computed at the observation point $(0,1 \mathrm{~m}, 0)$ for a static wheel load moving at different speeds have been plotted in Figs. 12-14. In contrast, the real-part displacement of the wheel load with a frequency of $f_{0}=10 \mathrm{~Hz}$ was plotted in Fig. 15. A comparison of Figs. 12 with 14 indicates that for the static load case, the responses are concentrated only in a very small duration, whereas for the case with a dynamic load, the responses oscillate and propagate for a rather long duration. Moreover, for the later case, a larger frequency of fluctuation is observed for the waves ahead of the arrival of the moving load than that following in the subsonic range. The two frequencies can be computed as

$f_{\mathrm{cr}}=f_{0} /\left(1 \pm \frac{c}{c_{\mathrm{R}}}\right)$

where $c_{\mathrm{R}}=92 \mathrm{~m} / \mathrm{s}$, according to the Doppler effect.

The effect of vibration frequency $f_{0}$ of the moving wheel load on the maximum (i.e. absolute) displacements, velocities and accelerations of the observation point was plotted in Fig. 16(a)-(c) with respect to the Mach number $M_{2}$. As can 
be seen, for the case with no self-oscillation, i.e. with $f_{0}=0 \mathrm{~Hz}$, there exists a distinct critical speed at $M_{2}=0.92$, but for the case with fluctuating wheel load, i.e. with $f_{0} \neq 0 \mathrm{~Hz}$, no distinct critical speeds can be observed. These results suggest that only when vibrations of the train wheels are neglected, can the critical region be determined for the train speed in relation to the R-wave speed of the soils. As a matter of fact, the critical speed for this case can be obtained from Eq. (35) as:

$c_{\mathrm{cr}}=\frac{ \pm\left(f-f_{0}\right)}{f} c_{\mathrm{R}}$

which can also be verified by the plot for the displacement $|\tilde{V}|$ vs. speed $c$ in Fig. 17 in frequency domain for $f_{0}=10 \mathrm{~Hz}$. For the case with $f_{0}=0 \mathrm{~Hz}$, the critical speed becomes equal to $c_{\mathrm{R}}$, in consistence with what we have observed. But for $f_{0} \neq 0 \mathrm{~Hz}$, the critical speed depends not only on $f_{0}$, but also on the frequency $f=\omega / 2 \pi$. However, as the time domain responses are computed as the superposition of the responses contributed by all the frequencies, the effect of critical speed can hardly be identified in time domain for the case with $f_{0} \neq 0 \mathrm{~Hz}$.

The other observation from Fig. 16 is that for $f_{0} \neq 0 \mathrm{~Hz}$, the influence of $f_{0}$ on the maximum displacements is quite different from its influence on maximum velocities and accelerations. In general, the increase of $f_{0}$ results in the decrease of the displacement, whereas the reverse is true for the velocity and acceleration. Moreover, for the cases with non-zero $f_{0}$, the higher the frequency of vibration $f_{0}$ of the moving load, the larger the magnitudes of the velocity and acceleration are. Nevertheless, it should be noted that all the results presented in Fig. 16 are based on the assumption that the amplitude of the wheel load remains constant, i.e. set equal to $T=10 \mathrm{t}$, regardless of the variation of the frequency $f_{0}$. For a moving static wheel load, this assumption is reasonable, but for a moving wheel load with non-zero $f_{0}$, the amplitude can be far less than the wheel weight $T$. Thus, for the case with nonzero $f_{0}$, the results presented herein serve merely as a qualitative illustration of the influence of the dynamic component of a traffic load on ground vibrations.

In order to investigate the effect of response attenuation, the vertical displacement, velocity and acceleration along a line which is located $1 \mathrm{~m}$ beneath the $x$-axis for a range of $0-20 \mathrm{~m}$ were plotted for loads moving in the subsonic speeds $(c=30$ $90 \mathrm{~m} / \mathrm{s})$ and transonic speeds $(c=110-170 \mathrm{~m} / \mathrm{s})$ with no oscillation, i.e. $f_{0}=0 \mathrm{~Hz}$, in Fig. 18. As can be seen, the attenuation trend is generally different for the subsonic and transonic speeds. For the subsonic case, the responses attenuate very quickly with respect to the distance, especially when the velocity and acceleration are concerned, whereas for the transonic case, the responses attenuate much more slowly. Besides, for the subsonic case, the response increases as the load speed increases, while for the transonic case, such a trend remains only true for the acceleration, but is reversed for the displacement and velocity.

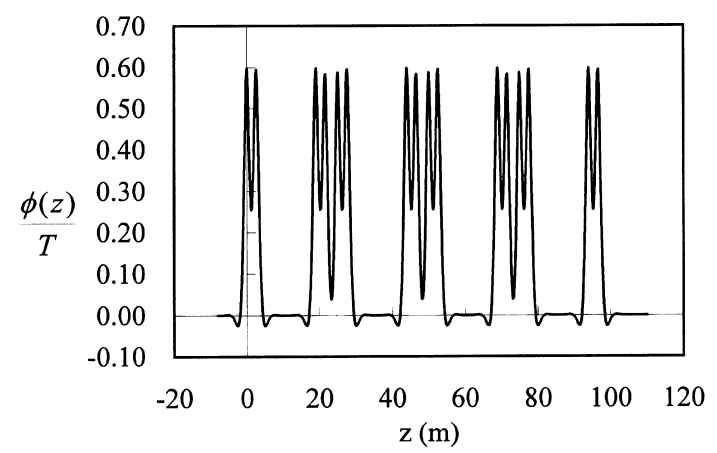

(a)

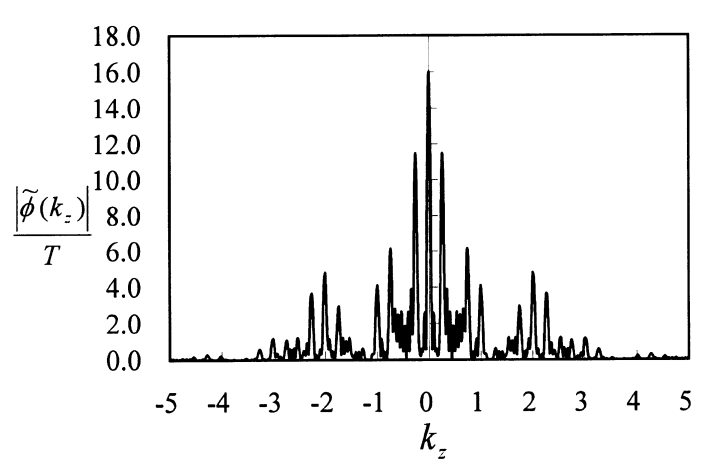

(b)

Fig. 20. A sequence of wheel loads: (a) load distribution function, (b) Fourier transform.

To investigate the attenuation behavior of the responses for moving loads with self oscillation, the vertical responses computed along the line located at $1 \mathrm{~m}$ beneath the $x$-axis induced by a wheel load moving at speed $c=70 \mathrm{~m} / \mathrm{s}$, but with different frequencies $f_{0}$, have been plotted in Fig. 19. As can be seen, the attenuation rates of responses for the case with zero $f_{0}$ are larger than that for the case with non-zero $f_{0}$. In general, the attenuation rate on the displacement response is higher for wheel loads with higher frequencies $f_{0}$ than that with lower frequencies.

\subsection{A Sequence of moving wheel loads}

As a final example, a moving load that takes into account the spatial distribution of the wheels of a real train will be considered. Based on the definition given in Eq. (31) and Fig. 3(b), the following dimensions are adopted: $a=2.56 \mathrm{~m}, b=16.44 \mathrm{~m}$ and $L=25 \mathrm{~m}$. The load distribution functions in the spatial and transformed domains for a carriage number of $N=4$ were shown in Fig. 20(a) and (b). In Fig. 20(b), the load distribution function in the transformed domain appears to be periodically distributed. Each peak in this figure relates to one passing frequency implied by the distances between wheels.

The effect associated with the number of carriages is investigated in Fig. 21, in which the maximum vertical 


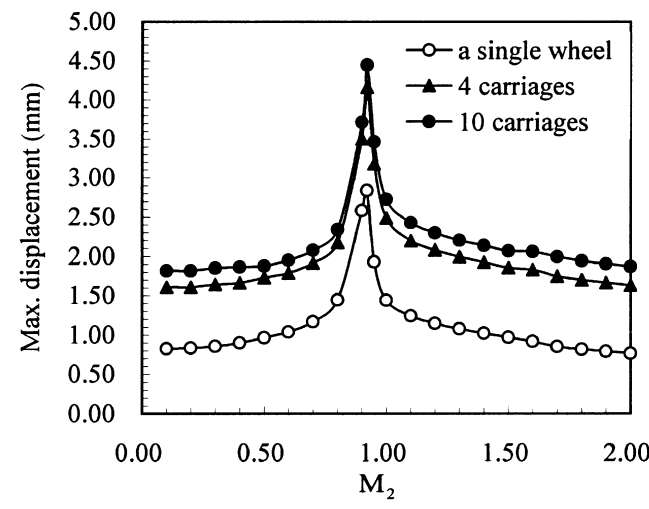

(a)

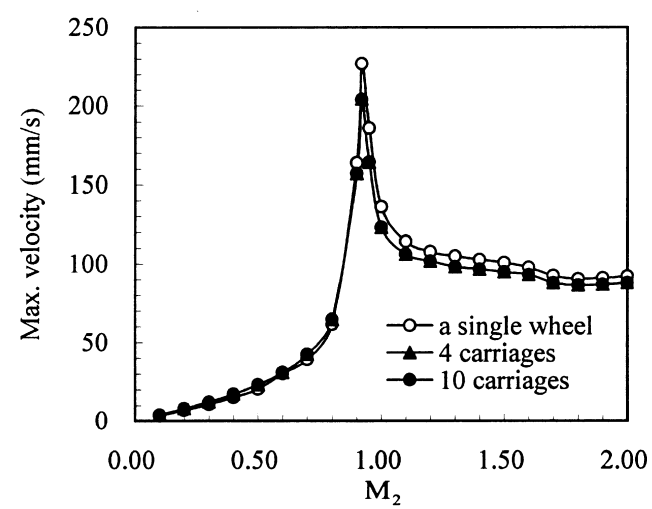

(b)

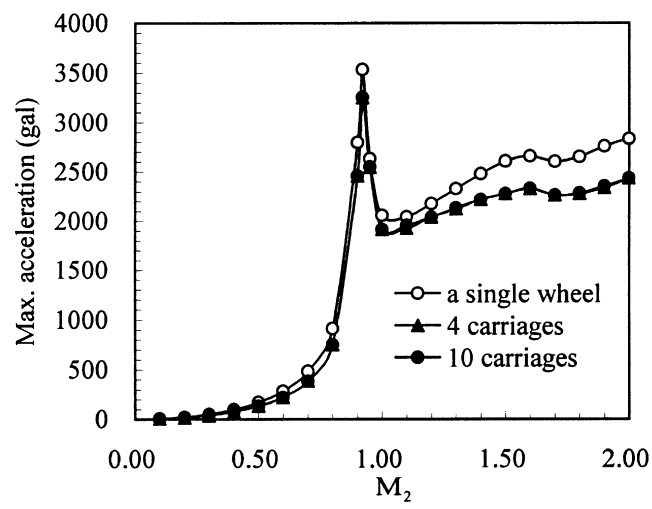

(c)

Fig. 21. Maximum vertical responses caused by a sequence of wheel loads: displacement, (b) velocity, (c) acceleration.

responses with respect to different Mach numbers $M_{2}$ were plotted for the three cases: a single moving wheel load, four carriages, and ten carriages. From Fig. 21(a), it is observed that with the increasing numbers of wheels or carriages, the displacement increases in all the speed range, but the difference between the cases with $N=4$ and $N=10$ is not so obvious. On the other hand, as can be seen from Fig. 21(b) and (c), basically no difference can be made for the velocity and acceleration responses as the carriage number $N$ increases from 4 to 10 , and the responses produced by a single wheel load appear to be even larger than the other two cases in the transonic and supersonic range.

\section{Concluding remarks}

In this paper, a procedure for computing the responses of a visco-elastic half-space subjected to various moving vehicle loads on the surface has been presented. The moving load is assumed to consist of a static part, to simulate the gravitational effect, and a dynamic part, to simulate the selfoscillation effect. Four different forms of load distribution functions are considered. The following conclusions can be drawn from the numerical studies conducted. (1) For the case with vertical moving loads, the critical speed is equal to the R-wave speed. (2) Unlike the case with static moving loads, the critical speed for a moving load with a fluctuating component depends on the particular frequency considered. Because of this, no obvious resonance phenomenon can be observed in time domain. (3) The dynamic component of a moving load can affect significantly the velocity and acceleration responses. As the fluctuating frequency increases, the amplitude of the velocity and acceleration increases as well. (4) The attenuation rate of the soil responses for moving loads traveling in the transonic and supersonic ranges is smaller than that in the subsonic range. (5) The velocity and acceleration of the soils induced by a moving load that vibrates itself with a higher frequency may attenuate faster than that with a lower frequency. (6) The increase in the number of carriages constituting a train may result in increase of the displacement, but not of the velocity and acceleration of the soils.

\section{Acknowledgements}

The research reported herein was sponsored partly by the National Science Council of the Republic of China through Grant No. NSC 89-2211-E-002-002.

\section{Appendix A.}

The following is a list of the entries involved in the [G] matrix of Eq. (16):

$$
\begin{aligned}
& g_{11}=k_{x}^{2}+k_{z}^{2}-\frac{1}{2} k_{\mathrm{S}}^{2} \\
& g_{12}=-i k_{x} m_{2} \\
& g_{13}=-i k_{z} m_{2} \\
& g_{21}=i k_{x} m_{1} \\
& g_{22} \frac{k_{z}^{2}}{m_{2}^{2}}\left(k_{x}^{2}+k_{z}^{2}-\frac{1}{2} k_{\mathrm{S}}^{2}-2 m_{1} m_{2}\right)+\left(k_{x}^{2}+k_{z}^{2}-\frac{1}{2} k_{\mathrm{S}}^{2}\right) \\
& g_{23}=k_{x} k_{z}\left[2 \frac{m_{1}}{m_{2}}-\frac{1}{m_{2}^{2}}\left(k_{x}^{2}+k_{z}^{2}-\frac{1}{2} k_{\mathrm{S}}^{2}\right)\right] \\
& g_{31}=-i k_{z} m_{1}
\end{aligned}
$$


$g_{32}=-k_{x} k_{z}\left[2 \frac{m_{1}}{m_{2}}-\frac{1}{m_{2}^{2}}\left(k_{x}^{2}+k_{z}^{2}-\frac{1}{2} k_{\mathrm{S}}^{2}\right)\right]$

$g_{33}=-\left[\frac{k_{x}^{2}}{m_{2}^{2}}\left(k_{x}^{2}+k_{z}^{2}-\frac{1}{2} k_{S}^{2}-2 m_{1} m_{2}\right)+\left(k_{x}^{2}+k_{z}^{2}-\frac{1}{2} k_{\mathrm{S}}^{2}\right)\right]$

\section{References}

[1] Eason G. The stresses produced in a semi-infinite solid by a moving surface force. International Journal of Engineering Sciences 1965;2:581-609.

[2] Gakenheimer DC, Miklowitz J. Transient excitation of an elastic half space by a point load traveling on the surface. Journal of Applied Mechanics, ASME 1969;36:505-15.

[3] Frýba L. Vibration of solids and structures under moving loads. Groningen, The Netherlands: Noordhoff International Publishing, 1972.

[4] Alabi B. A parametric study on some aspects of ground-borne vibrations due to rail traffic. Journal of Sound and Vibration 1992;153(1):77-87.
[5] de Barros FCP, Luco JE. Response of a layered viscoelastic halfspace to a moving point load. Wave Motion 1994;19:189-210.

[6] Luco JE, Apsel RJ. On the Green's functions for a layered half-space. Part I. Bulletin of Seismological of America 1983;73(4):909-29.

[7] Grundmann H, Lieb M, Trommer E. The response of a layered halfspace to traffic loads moving along its surface. Archive of Applied Mechanics 1999;69:55-67.

[8] Lieb M, Sudret B. A fast algorithm for soil dynamics calculations by wavelet decomposition. Archive of Applied Mechanics 1998;68:14757.

[9] Krylov V, Ferguson C. Generation of low frequency ground vibrations from railway trains. Applied Acoustics 1994;42:199-213.

[10] Krylov VV. Generation of ground vibration by superfast trains. Applied Acoustics 1995;44:149-64.

[11] Takemiya H. Prediction of ground vibration induced by high-speed train operation. 18th Sino-Japan Technology Seminar 1997, Chinese Institute of Engineers, 1-10

[12] Esveld C. Modern Railway Track. Duisburg, Germany: MRT-Productions, 1989.

[13] Piessens R, de Doncker-Kapenga E, Uberhube CW, Kahaner DK. QUADPACK. New York: Springer-Verlag, 1983.

[14] Fung YC. Foundations of solid mechanics. Englewood Cliffs, NJ: Prentice-Hall, 1965. 